\title{
Exploration of Intelligent Manufacturing Methods for Complex Products Driven by Multisource Data
}

\author{
Lei Zhang (iD \\ Electrical Engineering Department, Huaibei Vocational and Technical College, Huaibei 235000, China \\ Correspondence should be addressed to Lei Zhang; zhanglei1981@mail.sdu.edu.cn
}

Received 13 November 2021; Revised 13 December 2021; Accepted 23 December 2021; Published 12 January 2022

Academic Editor: Qiangyi Li

Copyright (c) 2022 Lei Zhang. This is an open access article distributed under the Creative Commons Attribution License, which permits unrestricted use, distribution, and reproduction in any medium, provided the original work is properly cited.

\begin{abstract}
In order to improve the multisource data-driven fusion effect in the intelligent manufacturing process of complex products, based on the proposed adaptive fog computing architecture, this paper takes into account the efficient processing of complex product intelligent manufacturing services within the framework and the rational utilization of fog computing layer resources to establish a fog computing resource scheduling model. Moreover, this paper proposes a fog computing architecture for intelligent manufacturing services for complex products. The architecture adopts a three-layer fog computing framework, which can reasonably provide three types of services in the field of intelligent manufacturing. In addition, this study combines experimental research to verify the intelligent model of this article and counts the experimental results. From the analysis of experimental data, it can be seen that the complex product intelligent manufacturing system based on multisource data driven proposed in this paper meets the data fusion requirements of complex product intelligent manufacturing.
\end{abstract}

\section{Introduction}

For traditional industries, it is undergoing technological changes. Industry 4.0 deeply integrates information technology and manufacturing and envisages a "smart factory" controlled by cyber-physical systems (CPS) [1]. Its main feature is the intelligence of information and the intelligence of production methods, including higher-level interconnectivity, smarter equipment, and more powerful data processing capabilities [2]. Through interconnected information, statistical data, and dynamic analysis, production can become smarter, leaner, more efficient, and more energy efficient. In the context of intelligent manufacturing, more and more objects are becoming intelligent, everything is interconnected, and the Internet of Things enters the factory. The Internet of Things is essential for data perception, collection, merging, transmission, and reverse control in smart factories [3].

In the decades, since the reform and opening up, my country has gradually formed a relatively complete industrial system and successfully ranked among the largest manufacturing countries in the world. However, under the current wave of global manufacturing development, factories in my country's manufacturing industry that realize automated production still account for a small number of factories, and most of the manufacturing enterprises have weak infrastructure and backward automation technology. In this "big but not strong" status quo, it is still a challenge to use more advanced technologies to replace the traditional production lines with low efficiency and high production labor costs. Moreover, in recent years, due to rising labor costs, social development transformation, and other needs, the improvement of manufacturing production technology has become increasingly urgent. In response to the urgent need for transformation and upgrading of the manufacturing industry, the Chinese government issued the "Made in China 2025" plan. It has come to focus on the development and promotion of the technological upgrading of the domestic manufacturing industry.

This study combines the multivariate data-driven method to study the intelligent manufacturing method of complex products and provides a theoretical reference for the intelligent manufacturing in the era of big data of the Internet of Things. 


\section{Related Work}

In the five to six years, since Industry 4.0 and fog computing were proposed, there have been some research studies on fog computing in the Industrial Internet of Things, most of which have been concentrated in the past three years, and theoretical research results are growing rapidly. Kuksa et al. [4] proposed that the main features of the Industrial Internet of Things are the shortage of communication resources caused by the growth of intelligently connected objects and special IoT data such as video streams, as well as the key implementation technologies related to fog computing. Finally, its performance is evaluated in a typical industrial scene. Stavroulakis and Papadimitriou [5] proposed a new SDN-based IoT architecture. Moradi and Zandi Paak [6] investigated and studied the computing and communication architecture in the Industrial Internet of Things. The Industrial Internet of Things architecture has three layers, smart object layer, fog computing layer, and cloud computing layer, and pointed out the computing and communication challenges faced in the Industrial Internet of Things which include unstructured data storage and computing requirements under the limit of edge network communication and the reasonable scheduling of services and traffic at the fog computing layer. Lazzeretti et al. [7] pointed out that one of the major challenges in fog computing in the Internet of Things are the use and release of resources. They proposed a fog computing architecture in the Internet of Things, including two layers of IoT and cloud computing, with cloud-fog middleware between the two layers. For communication, the fog computing belongs to the IoT layer. In addition, it has also optimized the two-tier resource allocation problem, reducing the delay by $39 \%$ and providing a reasonable fog resource allocation scheme for delay-sensitive applications. Akhmetshin et al. [8] proposed the best workload distribution method between fog as well as cloud. Constraint is with minimum power consumption as the optimization goal. Ugalde Hernández [9] uses approximate methods to solve the problem and reduces transmission delay. Isaksen and Trippl [10] study the QoS-awar allocation of the CFC, which meets the capacity demand at the same time. Hou et al. [11] proposed a cloud computing infrastructure, which includes the bottom layer, edge layer, and core layer. The bottom layer is the IoE (Internet of Everything) layer, the edge layer is the fog service layer, and the core layer is the cloud service layer [12]. Jones et al. [13] analyze the characteristics and problems of fog computing in Industry 4.0 and propose a fog computing framework based on the MQTT communication model and a machine learning algorithm for predicting the amount of data to reduce the data transmitted in the Internet of Things. Finally, this article uses real data sets to simulate the proposed data prediction algorithm, which has obvious effects compared to the traditional MQTT scheme. Guowu and Bai [14] consider the cloud and fog combination system in the Internet of Things, analyze the application characteristics of the IoT environment, and conduct modeling analysis for power consumption, service delay, carbon dioxide emissions, and cost. Experiment was conducted with traffic generated by the
100 most populous cities in eight geographically distributed data centers. The results show that, as the number of realtime service applications increases, the fog computing model is better than the traditional cloud computing model. For an environment, where $50 \%$ of applications request real-time services, the overall service delay time of fog computing can be reduced by $50.09 \%$. Liu et al. [15] studied the NP-hard problem of nondeterministic polynomials in the allocation of service requests. Taking the energy consumption and completion time of resource scheduling as the dual optimization goals, a heuristic-based resource allocation framework was proposed. The framework uses three heuristics. Formula algorithm, namely, particle swarm optimization, binary particle swarm optimization, and Bat algorithm.

\section{Multidata-Driven Fusion Algorithm for Intelligent Manufacturing of Complex Products}

Figure 1 is a block diagram of target state information fusion. The parameter data obtained from the multisensor output may be the azimuth angle (or pitch angle) of the target, the position and distance of the target in the observed platform, the speed of the target, etc. The fusion system performs effective data registration on the data from the sensor, converts it into a unified data expression form, then performs data association, and then obtains the estimation of the state vector. The literature has conducted strict mathematical evaluation and limitation analysis on the tracking fusion performance.

Data association is to assign the measurement of each sensor to its corresponding observation object. Once the observations of the same target are correctly associated, the estimation technology can get the target state. At present, the specific mathematical methods used for this level of fusion include Kalman filtering, sequential estimation method, joint probability data association (JPGA), multiple hypothesis method (MHT), and interactive multiple model method (IMM).

The target characteristic information fusion can be attributed to the problem of pattern recognition in essence. It is mainly used to extract more useful information about target characteristics from the observation information of multisensor than that of single sensor, so as to achieve more accurate classification and recognition of multitargets. The specific extraction process can use the same method for feature extraction and connect each group of feature vectors into a higher-dimensional feature vector. Moreover, it can also combine different methods to extract feature sets that cover more information and combine them into a new type of the feature vector. The fusion system fuses the target feature vector provided by each sensor and finally obtains the joint recognition result of the target identity. The specific process is shown in Figure 2.

The technologies for fusion recognition of targets mainly include feature compression and clustering algorithms, $K$ nearest neighbors, and neural network methods. The sensors 


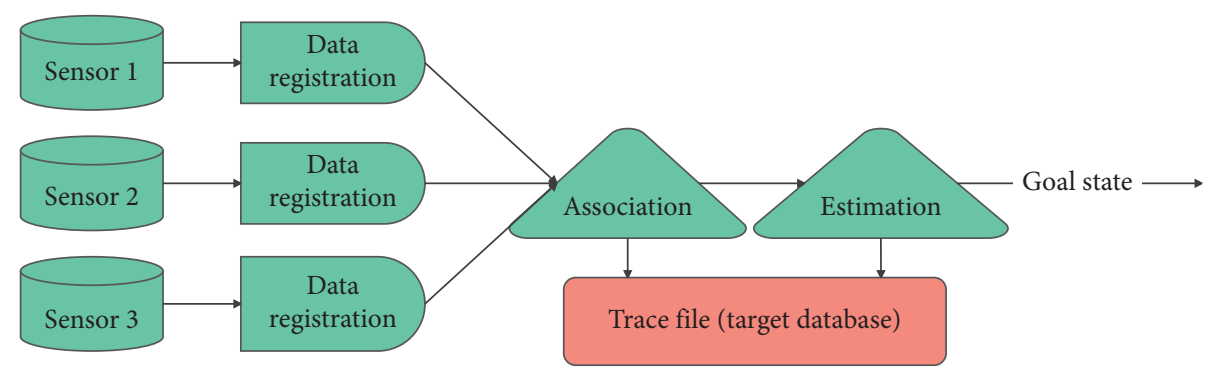

FIgURE 1: Block diagram of target state information fusion.

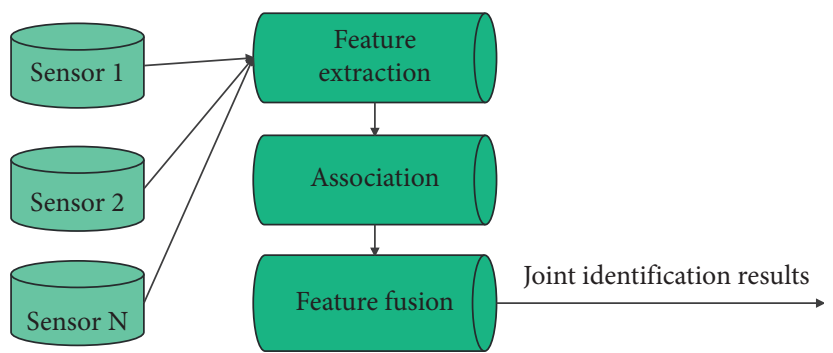

FIGURE 2: Block diagram of target characteristic information fusion.

participating in the fusion can be either a homogeneous sensor or a heterogeneous sensor so that it has greater flexibility.

The basic content of decision-making fusion is shown in Figure 3. The main process includes data preprocessing (such as unified data format), extracting relevant features, and identifying targets. After each sensor establishes a preliminary conclusion on the observed target locally, the central fusion module associates the previous conclusion and fuses the recognition results of each sensor to obtain the final joint inference result.

Decision-level fusion is a high-level fusion. Each sensor first makes intermediate decisions, and then, the fusion center completes the fusion processing of each intermediate decision. It can not only fuse information from heterogeneous sensors but also process asynchronous information with high flexibility. At present, expert system methods, Bayesian inference, Dempster-Shafer evidence theory, and other methods are often used in this level of fusion.

Kalman filter is suitable for linear systems with Gaussian noise distribution and can obtain the recursive minimum mean square error estimation of this type of system in the time domain. It has better filtering performance, and its iterative calculation is suitable for computer realization. The outstanding contribution of Kalman filtering lies in its successful introduction of the state space ideas in modern control theory into the optimal filtering theory, and the filtering is completed by constructing a dynamic model of the system. Moreover, it can not only handle stationary systems but also shows good filtering performance for timevarying systems and multidimensional, nonstationary signals. For decades, this classic filtering theory has been continuously deepened and developed in many research fields, and fruitful results have been achieved. In particular, it has been successfully applied in the field of target tracking and derived algorithms based on classical Kalman, such as extended Kalman filtering and insensitive Kalman. Since the standard Kalman filtering algorithm is the basis for solving the tracking problem and other extended or improved algorithms, the filtering process is briefly given here.

The state equation of the discrete-time system is

$$
x(k+1)=F(k) x(k)+G(k) u(k)+v(k) .
$$

The measurement equation of the discrete-time system is

$$
z(k+1)=H(k+1) x(k+1)+w(k+1),
$$

$w(k+1) \sim N(0, R(k+1))$. Moreover, it is assumed that the sequence of process noise and measurement noise has nothing to do with the initial state, and they are independent of each other.

In a filtering cycle, the initial state estimation and initial state covariance matrix $\hat{x}(0 \mid 0)$ and $P(0 \mid 0)$ are given [16].

The flowchart is shown in Figure 4 [17]:

We assume that the posterior probability density of the target state vector $x(k)$ at time $k$ is $p(x(k) \mid Z(k))$ :

$$
p(x(k) \mid Z(k)) \approx \frac{1}{N} \sum_{i=1}^{N} \delta\left(x(k)-x(k)_{i}\right) .
$$

The expectation of $x_{k}$ can be expressed as

$$
E[x(k)] \approx \int p(x(k) \mid Z(k)) \mathrm{d} x(k) \approx \frac{1}{N} \sum_{i=1}^{N} x(k)_{i} .
$$

In fact, the posterior probability density $p(x(k) \mid Z(k))$ is unknown, so it is not easy to directly sample particle samples from $p(x(k) \mid Z(k))$. The particles need to be sampled from a known importance density $q(x(k) \mid Z(k))$ that is convenient 


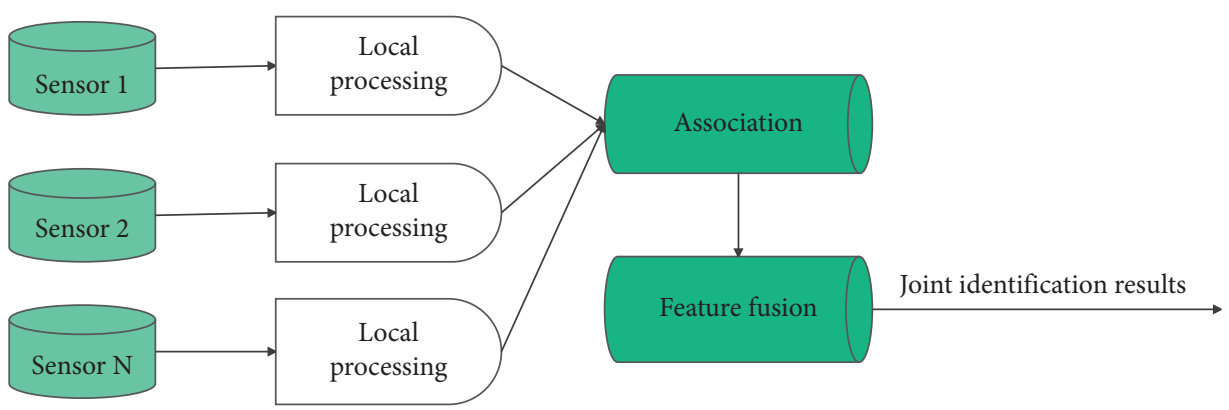

Figure 3: Block diagram of decision-making layer fusion implementation.

for sampling. Therefore, equation (12) is substituted as follows [18]:

$$
E[x(k)]=\int \frac{p(x(k) \mid z(k))}{q(x(k) \mid z(k))} q(x(k) \mid z(k)) \mathrm{d} x(k) .
$$

Equation (5) is used to substitute equation (4) as follows:

$$
E[x(k)]=\int \frac{w_{k}(x(k))}{p(Z(k))} \cdot q(x(k) \mid Z(k)) \mathrm{d} x(k) .
$$

Among them, the importance weight $w_{k}(x(k))$ is calculated as follows [19]:

$$
w_{k}(x(k))=\frac{p(x(k) \mid x(k)) \cdot p(x(k))}{q(x(k) \mid Z(k))} .
$$

By approximating the importance of equation [7], the following estimates are obtained [20]:

$$
\widehat{x}(k) \approx \frac{1}{N} \sum_{i=1}^{N} w_{k}\left(x(k)_{i}\right) \cdot x(k)_{i} .
$$

$w_{k}(x(k))$ can be estimated recursively as follows:

$w_{k}(x(k))=w_{k-1}(x(k-1)) \frac{p(z(k) \mid x(k)) \cdot p(x(k) \mid x(k-1))}{q(x(k) X(k-1), Z(k))}$.

We assume that the state estimate $\widehat{x}(k)$ of the target at time $k$ and the particle state effect $\hat{x}(k)_{i}$ is known, as well as the particle filter algorithm to recursively solve the estimated value $\hat{x}(k+1)$ of the target state vector when time is $k+1$, is as follows:

(1) For $i=1,2, \ldots, N$

$$
\begin{aligned}
& \text { Algorithm to } \\
& \widehat{x}(k+1)_{i} \sim q\left(x\left(k+1 \mid \widehat{x}(k)_{i}, z_{k}\right)\right)
\end{aligned}
$$

particles:

According to formula (9), the algorithm calculates the particle weight value $w_{i+1}^{k}\left(x(k+1)_{i}\right)$

End

(2) The algorithm calculates the total weight: $\operatorname{SUM}\left(w_{k+1}^{i}\left(x(k+1)_{i}\right)\right)_{i=1}^{N}$

(3) Regularized particle weight value: $\widetilde{w}_{k+1}^{i}\left(x(k+1)_{i}\right)=$ $w_{k+1}^{i}\left(x(k+1)_{i}\right) / \operatorname{SUM}\left(w_{k+1}^{i}\left(x(k+1)_{i}\right)\right)_{i=1}^{N}$

(4) The algorithm calculates the estimated value $\hat{x}(k+$ 1) of target state vector when time is $k+1$ according to formula (8);
(5) The algorithm performs resampling.

At that time, only the main ideas of JTC were initially explained. The joint target tracking and classification technology hopes to effectively and classification performance of the target through interaction between the target tracker and the target classifier. The basic idea is that the state estimator of the target (such as the target's motion characteristics and maneuverability reflected by the target's speed, acceleration, motion trajectory, etc.) can be used as the target's attribute characteristics to improve the target's classification performance. Correspondingly, the target attribute knowledge, such as target category information, helps to establish a more accurate target motion model, thereby improving target tracking performance. The basic principle of JTC is shown in Figure 5.

Since the introduction of JTC technology, a variety of related technical theories, such as Bayesian theory, D-S evidence theory, and fuzzy set theory, have been combined with the JTC technical framework to achieve target tracking and solve target recognition problems.

The state estimate of the target is

$$
E[\widehat{X}(k)]=\sum_{i=1}^{n} \int_{X(k)} p\left(X(k), c_{i} \mid Z(k)\right) \cdot X(k) \cdot \mathrm{d} X(k) .
$$

Traditional classic filtering algorithms are mostly based on a single model. Once the application scenario becomes more complex and the uncertainty increases, the dynamic characteristics of the target will change. Filters based on a single model often cannot match the changes in the target motion state in real time with a single model established in advance, which affects the effectiveness of tracking and even leads to divergence of the filter. As a result, the research proposed a multimodel tracking algorithm.

The multimodel tracking algorithm introduces the idea of self-adaptation into the target tracking algorithm. By establishing a set of possible motion modes of an object, each model in the model set represents a different motion mode. Then, each model is equipped with appropriate filters to perform parallel work, and the final state estimation output is the fusion result of the state estimation of each filter based on Bayesian inference. For example, the same target may run in the form of one or several combinations of uniform motion, uniform acceleration, and uniform turning motion during the entire movement. Using the multimodel tracking 


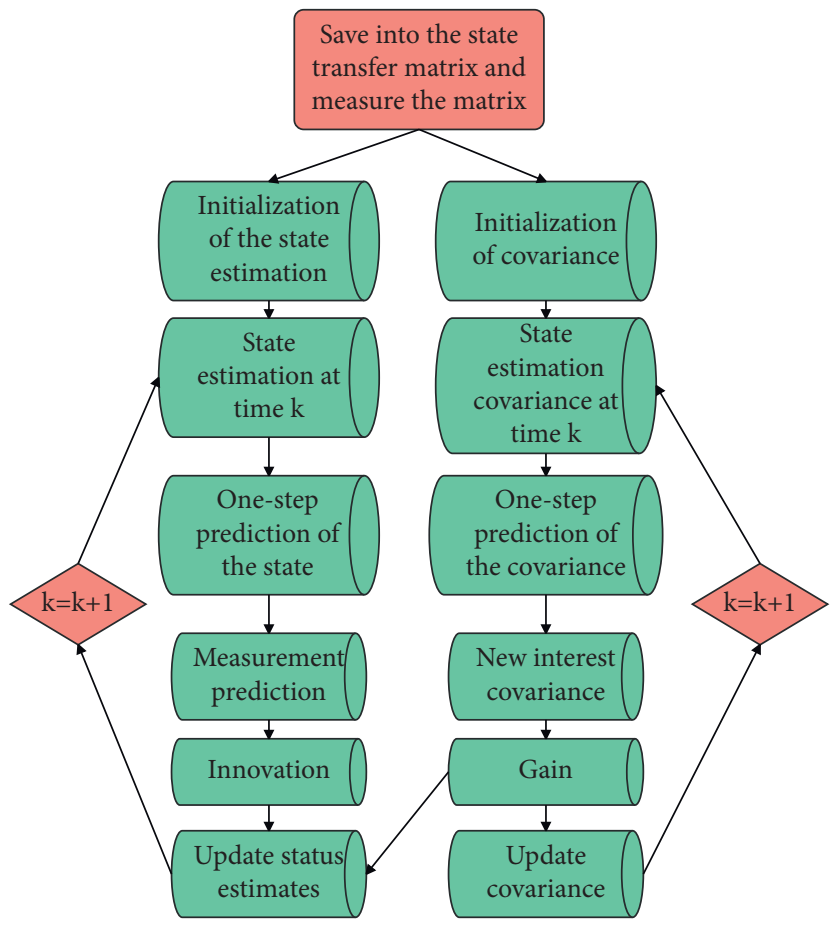

FIGURE 4: Flowchart of Kalman filter algorithm.

algorithm, the three models of CV, CA, and CT can be combined into multiple target operating modes to track them.

The implementation of the multimodel algorithm includes the following sections.

3.1. Design Target Motion Model Set. In order to apply the multimodel algorithm, it is necessary to design a model set consisting of a finite number of models based on certain prior knowledge in advance:

$$
M=\left\{m^{(j)}\right\}_{j=1,2, \ldots, r}
$$

Among them, each mode $m^{(j)}$ corresponds to one or more of the target motion modes under study. In the case of non-one-to-one correspondence, the model set is relatively simplified. In this case, the matching relationship can also be described as the system pattern at time $k$ is matched by the model $m^{(j)}$, that is,

$$
m_{k}^{(j)}=\left\{s_{j}=m^{(j)}\right\}, \quad k \in N, j=1,2, \ldots, r .
$$

The determination of the model set $M$ is an important basic work of the multimodel algorithm. From now on, we assume that all the system modes of the target motion can be combined and represented by the members in $M$.

3.2. Design of Filter. The algorithm selects some recursive filters suitable for each system mode in advance to complete the hybrid estimation. They can be linear filters or nonlinear filters as needed.
3.3. Estimated Fusion. The main task of estimation fusion is to process the results of each individual filter to complete the overall estimation. Commonly used estimation fusion methods include soft decision, hard decision, and random decision. Among them, soft decision (or no decision) is the mainstream method of multimodel estimation fusion. The overall estimate is the weighted sum of the probability of all filter estimates in the sense of least mean square:

$$
\widehat{x}_{k \mid k}=E\left(x_{k} \mid Z^{k}\right)=\sum_{i} \hat{x}_{(i)}^{k \mid k} P\left(m_{k}^{(i)} \mid Z^{k}\right) \text {. }
$$

3.4. Reinitialization of the Filter. Two basic multimodel algorithms are briefly given as follows: static multiple model (SMM) and deneralized pseudo-Bayesian estimator of $n$ order (GPBn).

In the SMM algorithm, multiple filters based on different models work independently and in parallel, regardless of the transition between models. The filters based on each model have no interaction with each other, and the target state estimation is the weight of the state estimation of each filter. The structure of the SMM algorithm is shown in Figure 6.

The so-called generalized pseudo-Bayesian method is that, at time $k$, only the history of the target model in the past finite sampling time interval of the system is considered when the system state is estimated.

The first-order GPB algorithm (GPB1) uses the simplest reinitialization method and only takes the overall state estimation $\hat{x}_{k-1 \mid k-1}$ at the previous moment and the covariance matrix $P_{k-1 \mid k-1}$ of the estimation error as the most common initial conditions. The respective state estimates are obtained through parallel filtering, and finally, the overall state 


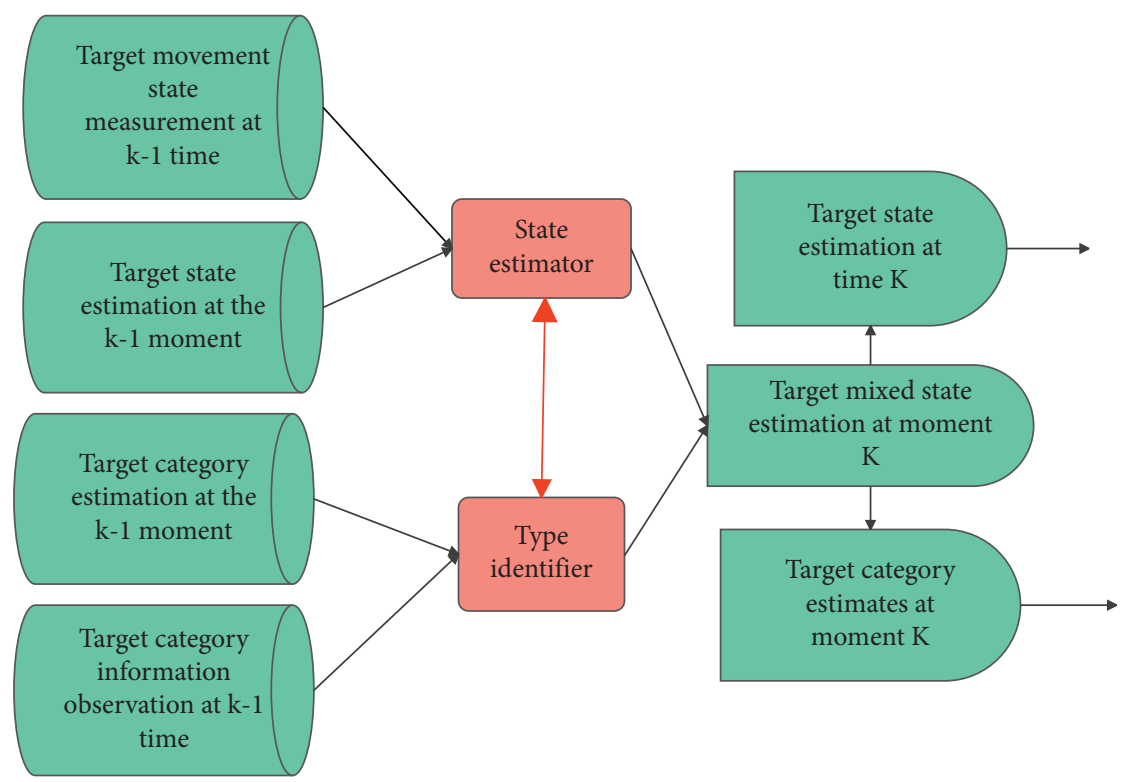

FIGURE 5: Schematic diagram of JTC algorithm framework.

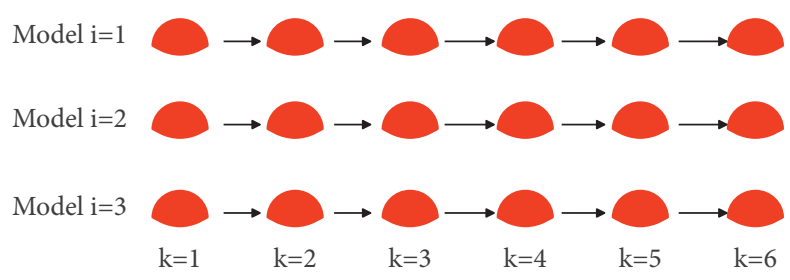

FIGURE 6: SMM algorithm structure diagram.

estimate at the current moment and the covariance matrix $P_{k \mid k}$ of the estimation error are obtained by the weighted sum method. The algorithm structure is shown in Figure 7.

The GPB1 algorithm assumes that the switching of the system mode between the elements of the model set satisfies the Markov property. The GPBn algorithm considers the model sequence of the most recent $n$ moments. The larger $n$ is, the more historical conditions that need to be considered, the larger the amount of memory occupied, and the more complex the algorithm is.

On the basis of GPB, an interactive multimodel algorithm (IMM) with Markov switching coefficient and a variable structure multimodel algorithm (VSMM) which is not limited to a priori model set were proposed in the later stage, which will not be introduced in detail here.

\section{Intelligent Manufacturing Method of Complex Products Driven by Multisource Data}

In the above section, this paper studies the multivariate datadriven algorithm and applies it to the research of intelligent manufacturing methods for complex products. The fog computing architecture of intelligent manufacturing services is shown in Figure 8.

MQTT is a message protocol based on the publish/ subscribe model of binary messages, which connects networks and devices with applications through middleware. The communication mode is divided into machine and server machine. MQTT has a lightweight, open, simple, standardized, and easy-to-implement design idea. It is very suitable for IoT scenarios and Industry 4.0. The application of MQTT in the Internet of Things is shown in Figure 9.

On this basis, this paper studies the multisource datadriven intelligent manufacturing system for complex products proposed in this paper, combines simulation research to verify the effect of multidata-driven fusion, and counts multiple sets of experimental data. The results are shown in Table 1.

From the above research, it can be seen that the system model proposed in this paper has a good multivariate data-driven fusion effect. On this basis, the effect of intelligent manufacturing management in this paper is evaluated, and the results shown in Table 2 are obtained.

From the above statistical results, the complex product intelligent manufacturing system based on 


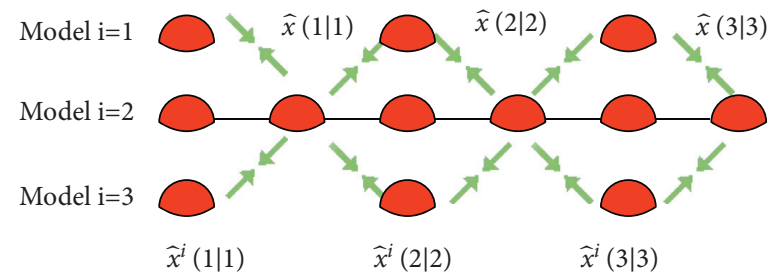

FIGURE 7: GPB1 algorithm structure diagram.
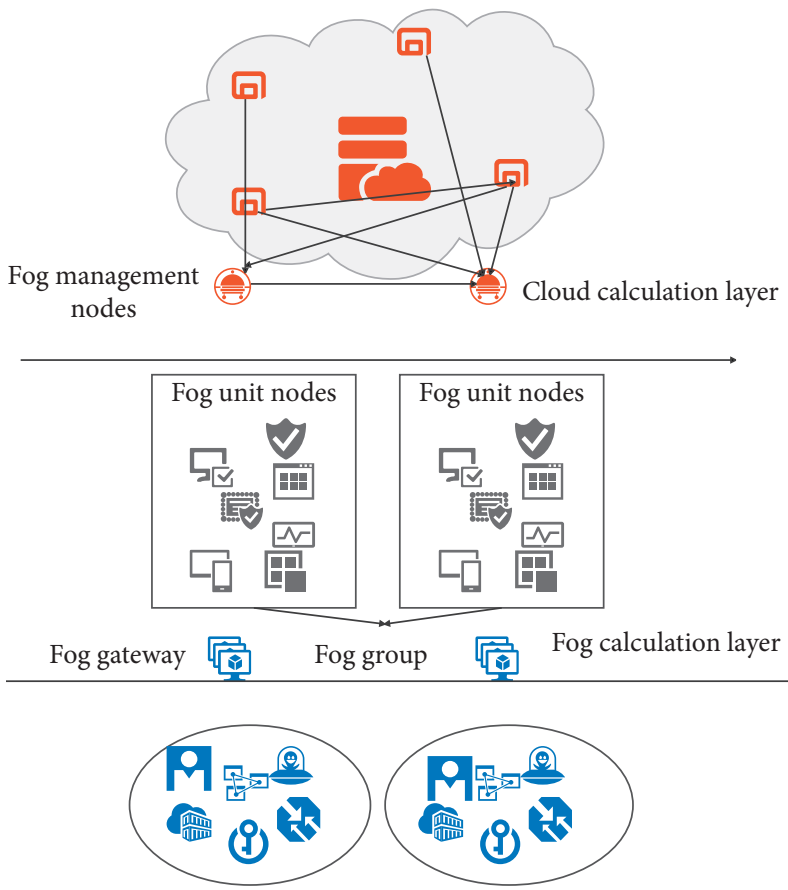

Comprehensive perception layer

FIGURE 8: Intelligent manufacturing system for complex products driven by multisource data.
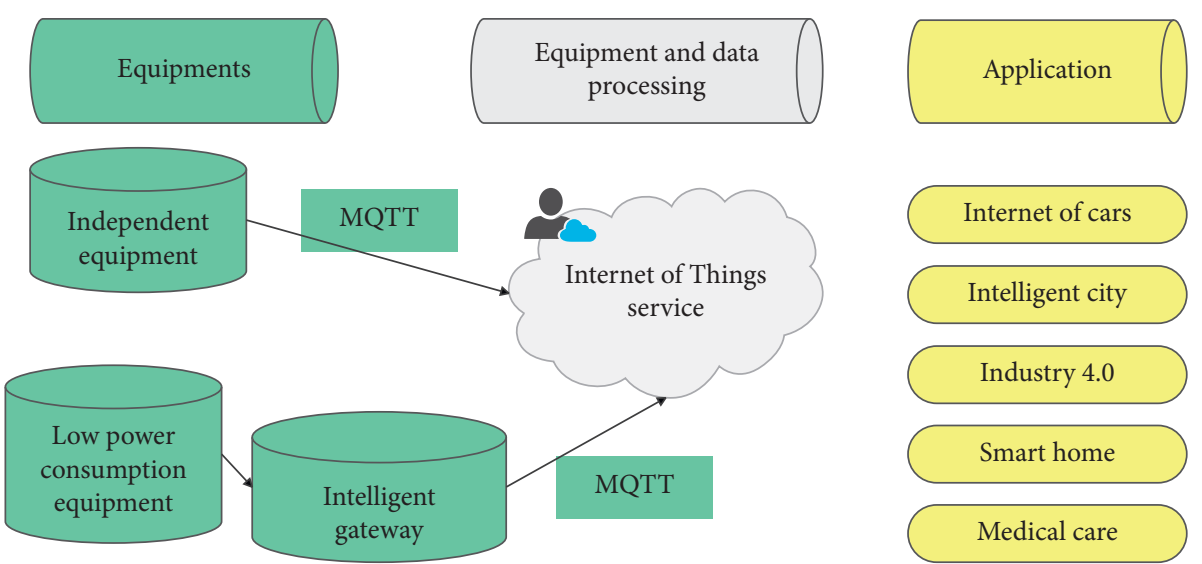

FIGURE 9: Application of MQTT in the internet of things. 
TABLE 1: Multivariate data-driven fusion effect.

\begin{tabular}{|c|c|}
\hline No. & Data fusion \\
\hline 1 & 90.81 \\
\hline 2 & 90.64 \\
\hline 3 & 86.45 \\
\hline 4 & 91.97 \\
\hline 5 & 90.78 \\
\hline 6 & 92.46 \\
\hline 7 & 89.35 \\
\hline 8 & 89.20 \\
\hline 9 & 90.04 \\
\hline 10 & 93.82 \\
\hline 11 & 87.71 \\
\hline 12 & 90.77 \\
\hline 13 & 92.00 \\
\hline 14 & 92.97 \\
\hline 15 & 93.37 \\
\hline 16 & 88.67 \\
\hline 17 & 87.82 \\
\hline 18 & 90.61 \\
\hline 19 & 88.51 \\
\hline 20 & 90.60 \\
\hline 21 & 87.98 \\
\hline 22 & 89.34 \\
\hline 23 & 90.05 \\
\hline 24 & 91.46 \\
\hline 25 & 93.96 \\
\hline 26 & 89.82 \\
\hline 27 & 87.10 \\
\hline 28 & 89.62 \\
\hline 29 & 92.23 \\
\hline 30 & 90.24 \\
\hline 31 & 87.35 \\
\hline 32 & 93.59 \\
\hline 33 & 93.41 \\
\hline 34 & 93.54 \\
\hline 35 & 88.00 \\
\hline 36 & 87.10 \\
\hline 37 & 86.77 \\
\hline 38 & 86.53 \\
\hline 39 & 92.44 \\
\hline 40 & 89.62 \\
\hline 41 & 89.43 \\
\hline 42 & 88.00 \\
\hline 43 & 87.36 \\
\hline 44 & 92.67 \\
\hline 45 & 86.61 \\
\hline 46 & 93.52 \\
\hline 47 & 90.43 \\
\hline 48 & 91.14 \\
\hline 49 & 91.62 \\
\hline 50 & 86.84 \\
\hline 51 & 88.29 \\
\hline
\end{tabular}


TABLE 2: Effectiveness of intelligent manufacturing management.

\begin{tabular}{|c|c|}
\hline No. & Smart manufacturing \\
\hline 1 & 88.59 \\
\hline 2 & 82.43 \\
\hline 3 & 87.19 \\
\hline 4 & 88.47 \\
\hline 5 & 91.65 \\
\hline 6 & 82.96 \\
\hline 7 & 91.70 \\
\hline 8 & 81.69 \\
\hline 9 & 87.57 \\
\hline 10 & 89.59 \\
\hline 11 & 91.17 \\
\hline 12 & 81.61 \\
\hline 13 & 87.69 \\
\hline 14 & 79.92 \\
\hline 15 & 82.18 \\
\hline 16 & 79.51 \\
\hline 17 & 82.66 \\
\hline 18 & 87.41 \\
\hline 19 & 83.95 \\
\hline 20 & 88.68 \\
\hline 21 & 88.19 \\
\hline 22 & 91.83 \\
\hline 23 & 79.86 \\
\hline 24 & 83.80 \\
\hline 25 & 83.24 \\
\hline 26 & 84.76 \\
\hline 27 & 88.16 \\
\hline 28 & 82.48 \\
\hline 29 & 85.97 \\
\hline 30 & 84.35 \\
\hline 31 & 84.29 \\
\hline 32 & 85.03 \\
\hline 33 & 89.48 \\
\hline 34 & 84.47 \\
\hline 35 & 82.01 \\
\hline 36 & 81.84 \\
\hline 37 & 85.83 \\
\hline 38 & 81.22 \\
\hline 39 & 87.60 \\
\hline 40 & 81.92 \\
\hline 41 & 83.30 \\
\hline 42 & 90.31 \\
\hline 43 & 84.80 \\
\hline 44 & 90.49 \\
\hline 45 & 85.69 \\
\hline 46 & 82.96 \\
\hline 47 & 87.24 \\
\hline 48 & 89.77 \\
\hline 49 & 84.34 \\
\hline 50 & 89.88 \\
\hline 51 & 79.48 \\
\hline
\end{tabular}

multisource data-driven proposed in this paper can play an important role in the intelligent manufacturing of complex products.

\section{Conclusion}

In the traditional way, the efficient processing of massive data can be realized by cloud computing. However, the networking situation is generally more complicated, the computing power of the underlying equipment is limited, and its transmission bandwidth and reliability have certain constraints. In addition, the Industrial Internet of Things also has the characteristics of large-scale connections and a large amount of communication data, and the application of intelligent manufacturing services is time sensitive. Because the devices in the Industrial Internet of Things require a lot of network and physical resources (in this paper, called fog resources) in order to perform different tasks in real time, at the same time, the ability of these fog resources to handle tasks is uneven. Therefore, how to effectively allocate these fog resources and how to efficiently cooperate with each other to obtain high efficiency and stability are the current challenges of the Industrial Internet of Things. This study combines multiple data-driven methods to study the intelligent manufacturing methods of complex products, which provide a theoretical reference for intelligent manufacturing in the era of big data in the Internet of Things.

\section{Data Availability}

The labeled dataset used to support the findings of this study are available from the corresponding author upon request.

\section{Conflicts of Interest}

The author declares no conflicts of interest.

\section{Acknowledgments}

The study was supported by "Key Natural Science Research Project of Anhui Universities in 2020" Research on Key Technologies of Large-sized THREE-DIMENSIONAL Precise Measurement Based on Multi-sensor Fusion (Grant no. KJ2020A0963), 2021 Excellent Youth Support Project in Anhui Universities (gxyqZD2021153).

\section{References}

[1] J. Novotná and L. Novotný, "Industrial clusters in a postsocialist country: the case of the wine industry in Slovakia," Moravian Geographical Reports, vol. 27, no. 2, pp. 62-78, 2019.

[2] A. A. Sozinova, O. I. Okhrimenko, L. V. Goloshchapova, N. Golovanova, and E. Tikhomirov, "Industrial and innovation clusters: development in Russia," International Journal of Applied Business and Economic Research, vol. 15, no. 11, pp. 111-118, 2017.

[3] M. Žižka, V. Valentová, N. Pelloneová, and E. Stichhauerova, "The effect of clusters on the innovation performance of enterprises: traditional vs new industries," Entrepreneurship and Sustainability Issues, vol. 5, no. 4, pp. 780-794, 2018.

[4] I. Kuksa, I. Shtuler, O. Orlova-Kurilova, I. Hnatenko, and V. Rubezhnaska, "Innovation cluster as a mechanism for ensuring the enterprises interaction in the innovation sphere," Management Theory and Studies for Rural Business and Infrastructure Development, vol. 41, no. 4, pp. 487-500, 2019. 
[5] P. J. Stavroulakis and S. Papadimitriou, "Situation analysis forecasting: the case of European maritime clusters," Maritime Policy \& Management, vol. 44, no. 6, pp. 779-789, 2017.

[6] M. Moradi and R. Zandi Paak, "The effect of companies' participation or non-participation in the creation of industrial clusters on their innovation performance with an emphasis on the mediatory role of knowledge management," Innovation Management Journal, vol. 5, no. 1, pp. 1-28, 2023.

[7] L. Lazzeretti, F. Capone, A. Caloffi, and S. R. Sedita, "Rethinking clusters towards a new research agenda for cluster research," European Planning Studies, vol. 27, no. 10, pp. 1879-1903, 2019.

[8] E. M. Akhmetshin, E. P. Kolpak, E. A. Sulimova, V. Kireev, E. Samarina, and N. Solodilova, "Clustering as a criterion for the success of modern industrial enterprises," International Journal of Applied Business and Economic Research, vol. 15, no. 23, pp. 221-231, 2017.

[9] O. Ugalde Hernández, "Local industrial agglomerations visà-vis global competitive networks: Marshallian notions of clusters, innovation and territorial development," Economía $Y$ Sociedad, vol. 25, no. 57, pp. 42-62, 2020.

[10] A. Isaksen and M. Trippl, "Innovation in space: the mosaic of regional innovation patterns," Oxford Review of Economic Policy, vol. 33, no. 1, pp. 122-140, 2017.

[11] B. Hou, J. Hong, H. Wang, and C. Zhou, "Academia-industry collaboration, government funding and innovation efficiency in Chinese industrial enterprises," Technology Analysis \& Strategic Management, vol. 31, no. 6, pp. 692-706, 2019.

[12] A. El Waatmani, "Industrial clusters and promotion of CSR: the case of developing countries," Journal of Academic Finance, vol. 9, no. 2, pp. 69-80, 2018.

[13] C. Jones, P. Pimdee, and P. Pimdee, "Innovative ideas: Thailand 4.0 and the fourth industrial revolution," Asian International Journal of Social Sciences, vol. 17, no. 1, pp. 4-35, 2017.

[14] L. Guowu and G. A. O. Bai, "Globalization and domestic coping strategies: the development of China's industrial clusters," Istanbul University Journal of Sociology, vol. 40, no. 2, pp. 625-647, 2020.

[15] Z. Liu, X. Chen, J. Chu, and Q. Zhu, "Industrial development environment and innovation efficiency of high-tech industry: analysis based on the framework of innovation systems," Technology Analysis \& Strategic Management, vol. 30, no. 4, pp. 434-446, 2018.

[16] F. Tödtling and M. Trippl, "Regional innovation policies for new path development-beyond neo-liberal and traditional systemic views," European Planning Studies, vol. 26, no. 9, pp. 1779-1795, 2018.

[17] M. Götz and B. Jankowska, "Clusters and Industry 4.0-do they fit together?” European Planning Studies, vol. 25, no. 9, pp. 1633-1653, 2017.

[18] J. Schot and W. E. Steinmueller, "Three frames for innovation policy: R\&D, systems of innovation and transformative change," Research Policy, vol. 47, no. 9, pp. 1554-1567, 2018.

[19] D. Chandrashekar and M. H. Bala Subrahmanya, "Exploring the factors of cluster linkages that influence innovation performance of firms in a cluster," Economics of Innovation and New Technology, vol. 28, no. 1, pp. 1-22, 2019.

[20] J. A. Belso-Martinez, I. Diez-Vial, M. J. Lopez-Sanchez, and R. Mateu-Garcia, "The brokerage role of supporting organizations inside clusters: how does it work?" European Planning Studies, vol. 26, no. 4, pp. 706-725, 2018. 